\title{
Construction of the Information Learning Platform for the Course of Concrete Structure Design Principle
}

\author{
Wenfeng Liu,Dehu Yu \\ College of Civil Engineering \\ Qingdao Technological University \\ Qingdao, 266033, China \\ lwf6688@sohu.com
}

\begin{abstract}
The course information learning platform is built on the modern information platform including digital technology, network communication technology and computer and multimedia technology and has become a new teaching mode. It changes the traditional mode of teaching and knowledge acquisition method, and also it provides the material platform for students' independent learning from the space-time limitation. During culture process of excellence engineers, it plays an important role in the coordinated development of the students' knowledge, qualities and abilities. Take the example of the course of concrete structure design principle, we discuss the important significance of information learning platform, development of multi-level teaching resources, design, construction and carry out the information learning platform for this course.
\end{abstract}

Keywords-Information Learning Platform;multi-level teachi ng resources; Teaching Website; Design; Implementation

\section{INTRODUCTION}

Course teaching is the most basic and fundamental level in higher education. In face of rapid technological development and application of digital multimedia networks, how to extend the multi-level teaching resources, how to build the Information Learning Platform for the courses, and how to formulate the new teaching model are the focus and difficulty in deepening the curriculum reform and the key to coordinating development of the students' knowledge, quality and ability at the course level.

With the development of modern science and technology, computer and network technology based distance education has become a necessary complement of the adult education model. It is built on the modern information platform including digital technology, network communication technology and computer and multimedia technology and has become a new teaching mode [1-2]. At present, various types of adult education have initiated the online courses construction work. Network courses may cross time, space and geographical boundaries and give adult learners the freedom to choose their learning time, space and environment. Thus ease the most prominent conflict between work and study in adult education. They prove to be conducive to the cultivation of the self-learning ability of adult learners. They promote changes in the way of learning and teaching, improve the information literacy of both teachers and students and provide more high quality online learning resources for lifelong education system and the formation of a learning society.

The course of concrete structure design principle is an important course for civil engineering specialty. The teaching quality, teaching method reform, teaching exploration and practice and experimental platform were studied in papers [3-6]. In this paper, we discuss the important significance of information learning platform, development of multi-level teaching resources, design, construction and carry out the information learning platform for this course.

\section{CONSTRUCTION OF INFORMATION LEARNING PLATFORM FOR COURSES}

\section{A. Research and development of multi-level teaching resources for different objects}

Learning material is the basis of the teaching resources. It includes text, images, animation, video and material in other forms. Among them, the text is the most widely used material and is also the foundation for building digital teaching resources. The textbook "Concrete Structure Design Principle" consists of a total of 460,000 words with all professional terms for each chapter translated into English. Based on the textbook, we have compiled an exercise guidance set of 230,000 words divided into 10 chapters. We have also compiled a sample test set of 180,000 words with 10 test samples ( 3 of which are online). Other text resources on the site are about 60,000 words, giving a total text resource of 930,000 words. The learning material also includes 226 AutoCAD drawings (non-scans) and a total of 3470MB (830 minutes) of teaching videos. We have also collected 35 animations and 12 teaching experimental videos. All of the above is the original creation of our research group.

Based on the basic material of text, formulas, charts, ani mation, video and other digital production, we have made PP T courseware consisting of 658 PPT pages and 226 pictures. PPT courseware has been converted into PDF format and dis played online(http://hnt.qtech.edu.cn/ppzy/hnt/dzja/).

We have digitized the printed material for students. Using VB source code, we have developed the CAI courseware (e-book). The courseware includes the e-learning materials, after-school exercises, self-test and knowledge development. It aims to meet the requirements of selflearning. The specialized vocabulary in each chapter has been translated into English, taking into account the bilingual 
teaching. The courseware contains a large amount of information with 812 pages and 202 pictures. The courseware has been made into a web page .

The electronic textbooks are designed based on students' learning needs. Each page includes a section title, the learning requirements and the teaching content of each chapter. Each professional term is provided with English equivalent. Each page contains view zooming, page turning and chapter switching functions. The drawing hand in the upper left corner of each page can control access to any page at any level. In program design, we have applied button design, toolbar production, form selection, animation design, multimedia calls, picture zooming, and other technologies. Take form selection as an example. We have applied MDI format. Each chapter of the courseware is a parent form with each section being a sub-form. The parent form is provided with menus and toolbars. The exercise part has the menu of editing, formatting, drawing, etc., each of them being set as a parent form. Each type of question forms a sub-form. The specific steps: Add MDI Form in the Project and set the startup form; set the property Windowstate $=2$, so that it covers the entire screen. Add Form and set MDIChild property to True; set Borderstyle $=2$ to automatically determine the size. Set MDIChild property of FORM1 using the standard form to True.

\section{B. Teaching Website Design and Implementation}

1) Teaching Website Design..Teaching website provides a material platform for students to study freely from the constraints of time and space. It is the integration of teaching ideas, design program, implementation strategy, and IT technology in network environment. It is the online extension of teaching.
The core of website design is teaching and learning in network environment. First is the instructional design. Teachers shall, based on teaching principles and requirements, design and construct websites which is the embodiment of teaching ideas and thoughts. Website design principles are teacher-led and student-centered. "Teacherled" is how to instruct, guide and encourage students. Based on the course websites, it is how to design and organize the effective knowledge (as knowledge is limitless in the open network), how to guide the learners, and how to carry out the teaching interaction. " Student-centered" means the students, free from the constraints of time and space, independently study, explore and research. Based on the course websites, it requires that the web content meet the needs of the students. Reasonable design ensures accessibility and availability. Second is the organization and structure of teaching resources. It is the core of the site. It includes courses outline and requirements, teaching resources, practical ability, and instructional interaction. The outline and requirements include course history, course syllabus, course content, teaching materials, teaching contents, teaching methods, and curriculum planning. Teaching resources include the full-range teaching videos, elearning materials, lesson plans, exercises guidance and sample tests. Practical ability includes course experiments, open experiments, scientific research, course paper, and construction competition. Interactive Teaching and Learning includes Q\&A through QQ and MSN, online Q\&A, online discussion, and teachers' thinking. Third, teaching evaluation and feedback. It includes school evaluation, supervisor evaluation, student evaluation, and the evaluation of internal and external experts. Through the operation of the site, we may constantly sum up experience and improve the site.

Design and Architecture of Course Website for "Concrete Structue Design Principle"

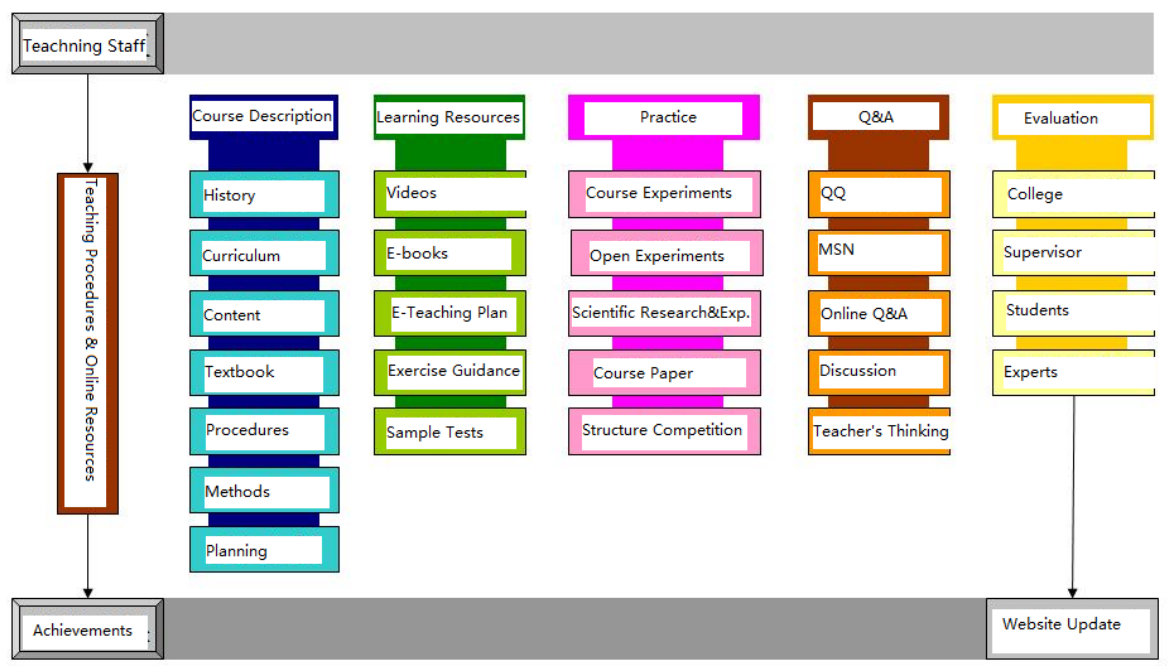

Figure 1. Website Design and Architecture 
2) Teaching Website Implementation. Website implementation is the integration of instructional design with modern teaching technology. The website is powered by the LAMP stack, Linux operating system, Apache Web services platform, My SQL open source database, and PHP scripting language. PHP requests sheet data and database information through URL. The Apache can let the user access to web pages. Website users may log on pages at any time, actualizing online learning. Different types of teaching resources including pictures, animation, PDF, and videos are nested with html tags through the invocation of the PHP function, displaying the required teaching resources on a web page. The following is program for the invocation of the image's PHP functions and the sequence of html tags of animation, PDF, and WORD.

PHP function of the image information getimageinfo (): $<$ ?php function getimageinfo (img) $\{/ /$ img is the absolute path to the image file

img_info = getimagesize (img);

switch (img_info [2]) \{

case 1:

imgtype = "gif";

break;

case 2:

imgtype = "jpg";

break;

case 3:

imgtype = "png";

break;

\}

img_type = imgtype. "Image ";

Get the file size

img_size = ceil (filesize (img) / 1000). "k"; / /

$$
\begin{aligned}
& \text { new_img_info = array ( } \\
& \text { "Width" => img_info [0], } \\
& \text { "Height" => img_info [1], } \\
& \text { The "type" => img_type } \\
& \text { "Size" => img_size } \\
& \text { \} } \\
& \text { return new_img_info; } \\
& \text { \} } \\
& \text { ?> }
\end{aligned}
$$

Animation and video animation invoke the <embed> tags:

Properties:

embed the src $=$ url

Description: The embed can be used to insert a variety of multimedia. The format can be Midi, Wav, AIFF, AU and MP3. Netscape and the new version of IE both support them. Url is the audio or video file and their path, being either relative or absolute.

\author{
Demo: <the EMBED SRC = "001.mid" \\ HIDDEN $=$ TRUE AUTOSTART $=$ TRUE \\ LOOP $=$ TRUE $>$ \\ PDF, Word and other formats directly use $<\mathrm{a}>$ tag: \\ Properties: \\ Href $=$ url
}

Description: a tag can be used to insert various contents not ready for direct display, such as PDF and Word. Url is the file path, being either absolute or relative.

Demo: <a herf $=$ "/ the file./ 01 /large eccentric compression experiment demonstration.

$$
\text { Doc " }></ \text { a }>
$$

3) Website Profile. Considering the length of this paper, here we only introduce the Home Page and the main Teaching Resources pages.

Home page is the heart of the website. It shall reflect the needs and cognition of students and other users and requires a deep understanding of the role of video, animation, pictures, text and various types of teaching resources on the students' cognitive level. It shall reasonably and effectively organize teaching resources and allow students and other users to gain fast access to teaching resources to achieve the goal of the network learning. The website, in accordance with the needs of students, provides both home page and navigation bar. The home page access contains 3 secondlevel pages and 16 third-level pages. The navigation bar access contains 11 second-level pages and 126 third-level pages. There are 74 third-level pages under the exercise guidance page (second-level). Site page is of beautiful design with rich teaching resources (http://hnt.qtech.edu.cn/ppzy/hnt/ ).

Based on cognitive rules and research, we place the most important course information and students' most wanted information on the home page. Access is actualized through a super link. The home page contains 5 secondlevel pages (two of them can also be accessed through the navigation bar) and 16 third-level pages, covering the course history, course description, characteristics of curriculum, course content, curricula, textbooks, reference material and educational videos of 9 chapters. The addresses of the second-level pages are as follows:

Course outline: http://hnt.qtech.edu.cn/ppzy/hnt/kcgk/ ;

Course content: http://hnt.qtech.edu.cn/ppzy/hnt/sh/ ;

Teaching video: http://hnt.qtech.edu.cn/ppzy/hnt/luxia ng/

The navigation bar access, according to the type and nature of the teaching resources, is divided into different levels, ensuring a step by step access into the pages. It is placed in the upper part of the home page, ensuring ready accessibility for students. The levels of the navigation bar are as follows. 


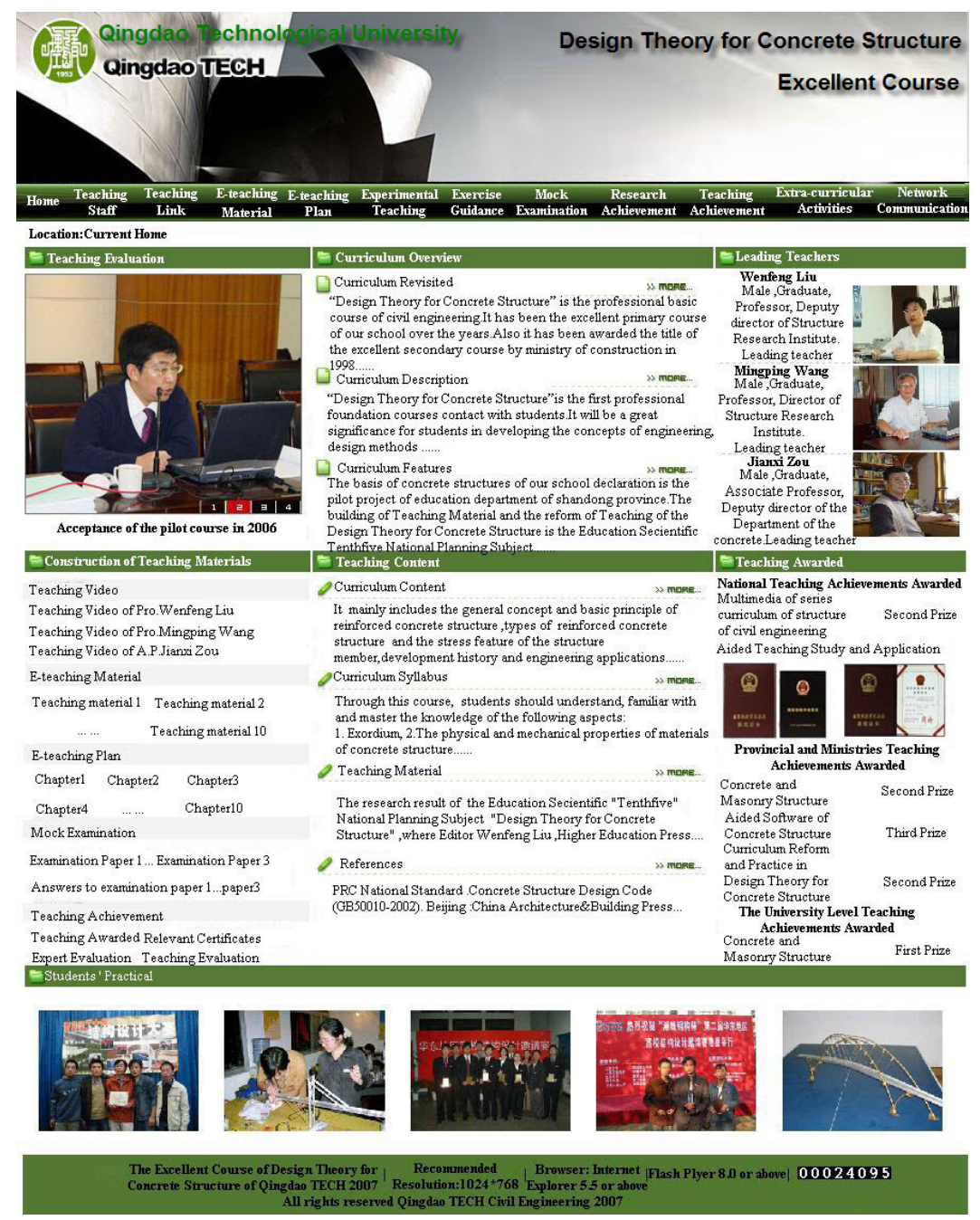

Figure 2. Website Home Page

The addresses of the second-level pages through the navigation bar are as follows:

Teaching staff: http://hnt.qtech.edu.cn/ppzy/hnt/szdw/ ;

Teaching content: http://hnt.qtech.edu.cn/ppzy/hnt/jxhj/ ;

E-learning materials: http://hnt.qtech.edu.cn/ppzy/hnt/dzjc/ ;

Electronic lesson plans: http:/hnt.qtech.edu.cn/ppzy/hnt/dzja/;

Experimental teaching,: http://hnt.qtech.edu.cn/ppzy/hnt/syjx/;

Exercise guidance: http://hnt.qtech.edu.cn/ppzy/hnt/xtzd/

Sample tests: http://hnt.qtech.edu.cn/ppzy/hnt/mnks/ ;

Teaching and research achievements: http://hnt.qtech.ed u.cn/ppzy/hnt/yjcg/ ;

Teaching Achievements: http:/hnt.qtech.edu.cn/ppzy/hnt/jxcg/;

Extracurricular activities: http:/hnt.qtech.edu.cn/ppzy/hnt/kwh $\mathrm{d} /$;

Onlie Q\&A: http://hnt.qtech.edu.cn/ppzy/hnt/wljl/
The website teaching resources include electronic lesson plans, e-learning materials, teaching videos, exercise guidance, and sample tests. Students may complete the whole process of study, practice and test online. As the electronic lesson plans and e-learning materials have already been introduced before, we will not repeat there. The instructional videos are completed by Wang Mingping, Liu Wenfeng, Zhu Yingjie, Zou Jianxi, Gao Litang and Guo Peng (6 teachers respectively of old, middle, and young ages). The videos cover nine chapters (one chapter for selfstudy), totaling $3470 \mathrm{MB}$ and 830 minutes. The detailed information are as follows: the exercise guidance set contains 10 chapters, including T/F questions, multiplechoices, essay questions and calculation problems. All are provided with answers, totaling 230,000 words. Furthermore, there are 3 sample tests and their answers. 


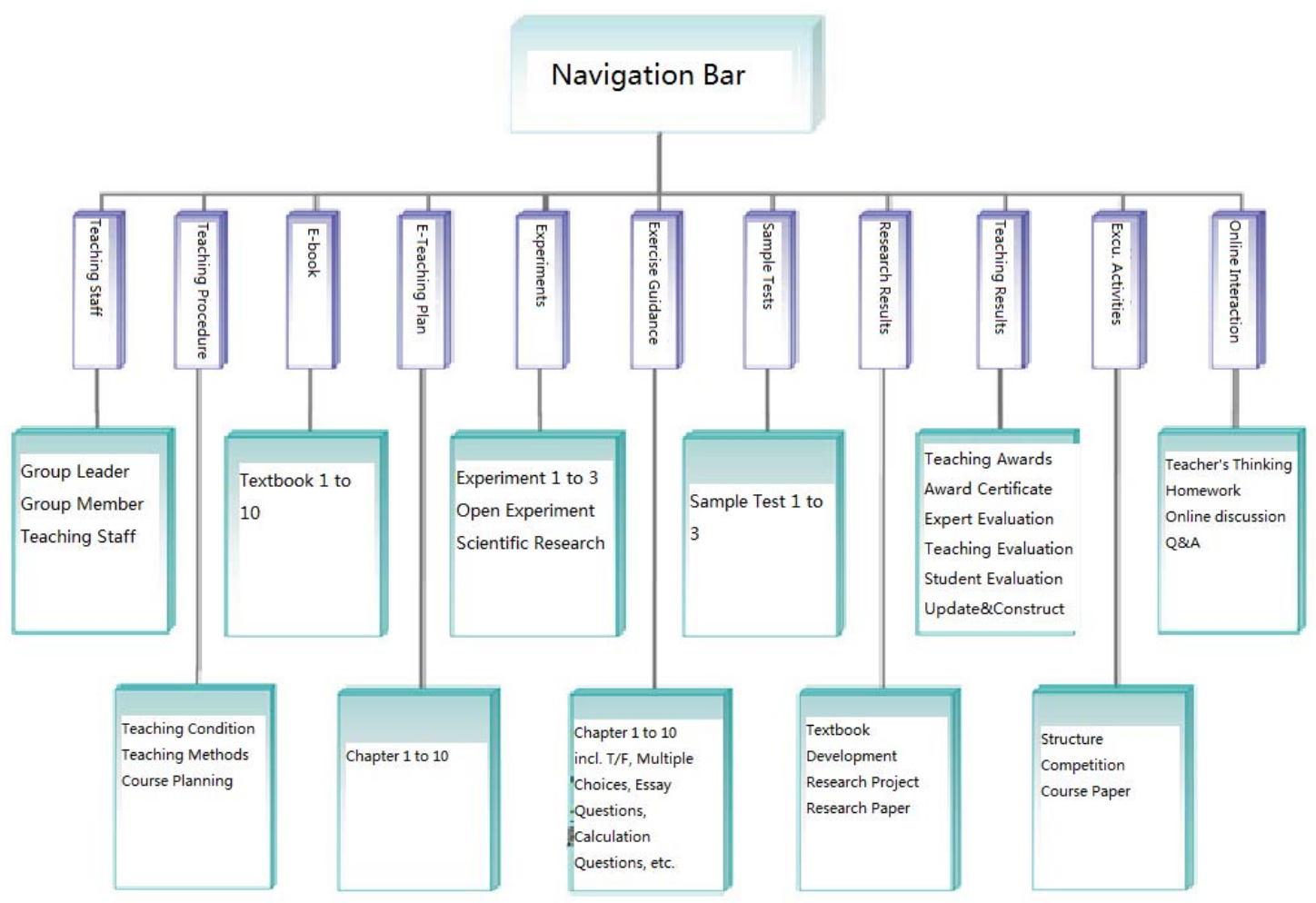

Figure 3. Hierarchical Structure of the Website Navigation Bar

\section{CONCLUSIONS}

Practice has proved that the construction of Information Learning Platform must be teacher-led and student-centered. And its fundamental purpose is to effectively improve students' professional ability, practical ability and innovation capacity. Thus ensure the vitality of the Information Learning Platform. In accordance with the precepts of education and teaching and combining the features of the Internet, the Information Learning Platform integrates syllabus, teaching arrangements, e-learning materials, electronic lesson plans, teaching videos, exercise guidance, sample tests, experiment guidance, online Q\&A, discussion, teachers' thinking, and reference material. It provides a material platform for students regardless of time and space restrictions. The course of "Construction of Concrete Structure Design Principle" has been awarded with the Quality Course in Higher Education in Shandong Province and has been recommended by Shandong Provincial Department of Education to participate in the competition for the national quality courses for two consecutive years.

\section{ACKNOWLEDGMENT}

This paper is one of the research results of the National Engineering Education Excellence Project of Qingdao Technological University, the National Special and Professional Project, and the Special and Professional Project in Shandong Province.

\section{REFERENCES}

[1] Li X L,Yan D. 2008 J. China Educational Technology.6 58

[2] Sun Y,Hu S W and Li G B.2011 J Distance Education in China.12 45.

[3] Gu X L,Lin F,Huang Y J,Zhao Y and Wang W. 2009 J.Research and Exploration in Laboratory.2 42.

[4] Cai J,Chen Q J and Huang Y S.2008 J.Journal of Architectural Education in Institutions of Higher Learning.5 70.

[5] An R M,Duan SH J,2007 J.Higher Education Forum.3 79.

[6] Pan Y.2011 J,Journal of Architectural Education in Institutions of Higher Learning.4 78. 